\title{
GCU
}

Glasgow Caledonian

University

University for the Common Good

\section{A single chip solution for pulse transmit time measurement}

Knezevic, Sasa; Stojanovic, Radovan; Asanin, Bogdan; Karadaglic, Dejan

Published in:

2013 IEEE 13th International Conference on Bioinformatics and Bioengineering (BIBE)

DOI:

10.1109/BIBE.2013.6701625

Publication date:

2013

Document Version

Author accepted manuscript

Link to publication in ResearchOnline

Citation for published version (Harvard):

Knezevic, S, Stojanovic, R, Asanin, B \& Karadaglic, D 2013, A single chip solution for pulse transmit time measurement. in 2013 IEEE 13th International Conference on Bioinformatics and Bioengineering (BIBE)., 14026341, IEEE, pp. 1, 13th IEEE International Conference on Biolnformatics and BioEngineering, Chania, Greece, 10/11/13. https://doi.org/10.1109/BIBE.2013.6701625

\section{General rights}

Copyright and moral rights for the publications made accessible in the public portal are retained by the authors and/or other copyright owners and it is a condition of accessing publications that users recognise and abide by the legal requirements associated with these rights.

Take down policy

If you believe that this document breaches copyright please view our takedown policy at https://edshare.gcu.ac.uk/id/eprint/5179 for details of how to contact us. 


\title{
A Single Chip Solution for Pulse Transmit Time Measurement
}

\author{
Saša Knežević, Radovan Stojanović, Bogdan Ašanin and Dejan Karadaglić
}

\begin{abstract}
This paper proposes a methodology for Pulse Transmit Time measurement for purposes of cuff-less monitoring of Blood Pressure. The ECG and PPG signals are simultaneously acquired and Pulse Transmit Time is determined as a time between $R$ peak in ECG and maximal slope in PPG. To distinguish characteristic points Discrete Wavelet Transform, Derivative Filtering, Modulus Maxima and additional functions are employed and translated in VHDL code and then embedded in a single chip from FPGA technology. The system works on-line, achieving accuracy of $97.53 \%$ and $97.09 \%$ for $R$ peak and slope detection respectively. The emphasis is given to the description of signal processing approach, system architecture and working principles of fundamental components. Also, the preliminary testing results are presented.
\end{abstract}

\section{INTRODUCTION}

$\mathrm{S}_{\mathrm{B}}$ upplemented by other physiological parameters, the Blood Pressure (BP) is considered as an invaluable diagnostic parameter to access the condition of certain illnesses, especially cardiovascular, which are a leading cause of death worldwide [1]. Continuous monitoring of BP is highly recommended in order to respond timely. The measurement techniques are divided into two categories: direct and indirect. The direct technique is in fact invasive, used when a high level of accuracy, dynamic response and continuous monitoring are required. A catheter with pressure transducer is directly inserted into blood vessel. The indirect techniques, almost existing more than one century, rely on concept of inflatable cuff to the arm which applied auscultatory or oscillometry principle. Presence of the cuff reduces the efficiency and flexibility of the meters in terms of power consumption, measurement frequency, patient mobility, ease of use, etc.

The Pulse Transit Time (PTT) method is a cuff-less alternative to the classical indirect methods [2]. The PTT is the time which BP pulse takes to travel from one arterial site to another. It is inverse proportional to the $\mathrm{BP}$ value. Typically, the PTT is initiated by the R-wave of the ECG signal and the arrival, at finger or ear, is, usually, detected

Manuscript received August 21, 2013. This work was supported by National project of Ministry of Science of Montenegro, MESI, and EU TEMPUS project BioEMIS. The authors are thankfull for support.

$\mathrm{S}$. K. is with the Faculty of Electrical Engineering, University of Montenegro, Podgorica, Montenegro (e-mail: sasaknezevic@live.com)

R. S. and N.L. are with the Faculty of Electrical Engineering, University of Montenegro, Podgorica, Montenegro (e-mail: stox@ac.me).

B.A. is with the Faculty of Medicine, University of Montenegro, Podgorica, Montenegro (e-mail: basanin@ac.me).

D. K. is with the School of Engineering and Built Environment, Glasgow Caledonian University, Glasgow, United Kingdom (e-mail: Dejan.Karadaglic@gcu.ac.uk). from photoplethysmograph (PPG) signal, in a point of its maximal slope MS, Fig. 1.

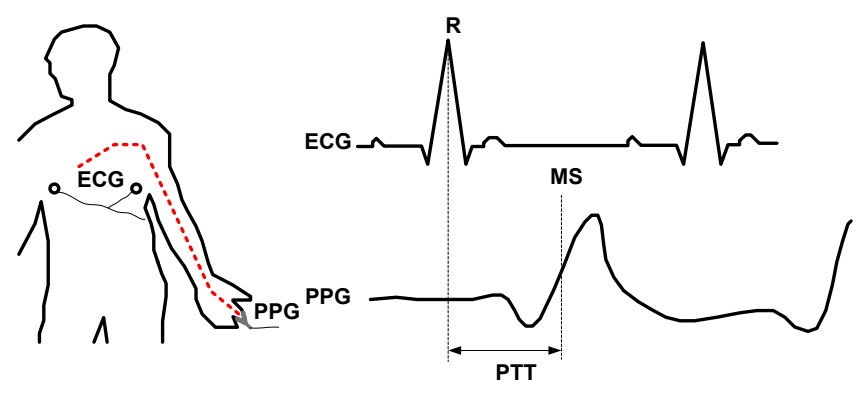

Fig. 1. The principle of PTT measurement.

The suspension of cuff and beat-to-beat tracking are the main advantages that recommend this method for application in wearable and telemedical devices, which are autonomous, miniature, low-cost, ultra-low-power devices and very often in form of a single chip. In practice, it is not a trivial task to embed all necessary processing operations in a single chip of limited arithmetic, memory and power consumption performances. An additional request is on-line operation.

This paper presents a trial in this direction. It proposes a methodology to implement an on-line system for PTT measurement in a single chip from FPGA technology. First, the characteristic points in ECG and PPG signals are distinguished using optimized Discrete Wavelet Transform (DWT), Derivative Filtering, Modulus Maxima and additional functions. Second, all signal processing functions are developed in VHDL code and embedded into functional modules which are later integrated in FPGA chip. Third, the approach is verified using real signals and obtained results are presented and discussed.

\section{Methodology}

Signal processing and operation flowchart consists of two parallel tracks: QRS detection and PPG slope detection, Fig. 2. Initially, the both signals are passed through the DWT decomposition module which consists of low-pass (L) and high-pass (H) filters according to the Mallat's scheme [3] $A_{i}(n)$ and $D_{i}(n)$, known as approximation and detail coefficients, are the outputs from these filters for $i^{\text {th }}$ decomposition level. In this research the integer modification of Haar wavelet transform, here marked as Haar DWT, is used for wavelet decomposition [4].

In case of ECG, Haar DWT, as any other wavelet transform, is capable to distinguish the QRS complexes within ECG signal. The $D_{i}(n)$ coefficients across the scales show that the R peak of the QRS complex corresponds to the 
zero crossing (ZC) between two local modulus maxima. In case of PPG, the approximation coefficients $A_{i}(n)$ are fed to the first order derivative filter producing signals $\operatorname{Dif}_{i}(n)$. Maximal slope in original PPG signal, MS, corresponds to the local maxima in $D 1 f_{i}(n)$. To improve accuracy of detection the coefficients $\mathrm{D} 2 \mathrm{fi}(n)$ and $D 3 f_{i}(n)$ are also examined on local maxima. When R or MS are detected the adequate trigger signals are generated and forwarded to the PTT calculator module, which is in fact a timer for PTT measurement.

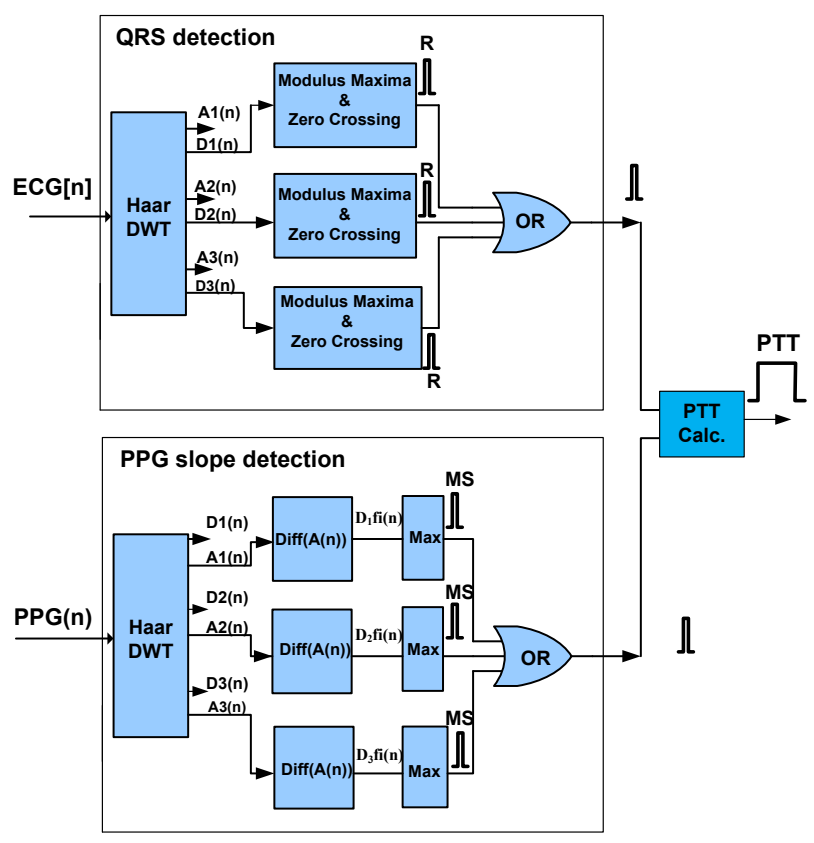

Fig. 2. PTT Signal processing approach.

Fig. 3 illustrates the decomposition of real discrete signal $E C G(n)$ up to the $4^{\text {th }}$ decomposition level of details, $D_{1}(n)$, $D_{2}(n), D_{3}(n)$ and $D_{4}(n)$. Simultaneously the signal PPG(n) is decomposed till $4^{\text {th }}$ level of approximations, $A_{1}(n), A_{2}(n)$, $A_{3}(n)$ and $A_{4}(n)$ and then fed to the derivative filter, producing signals $D 1 f_{i}(n), D 2 f_{i}(n), D 3 f_{i}(n)$ and $D 4 f_{i}(n)$. It is seen how R peak corresponds to the zero crossing of $D_{i}(n)$ and MS to local maxima of $D 1 f_{i}(n)$.

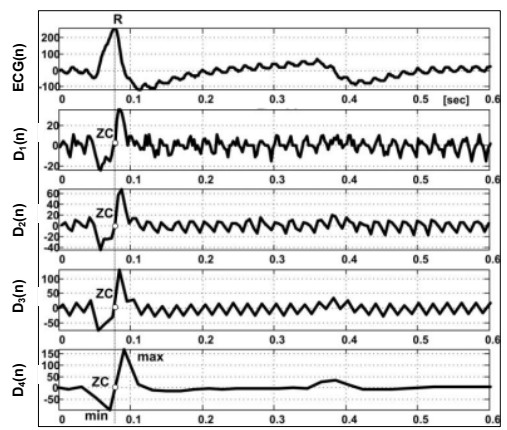

a)

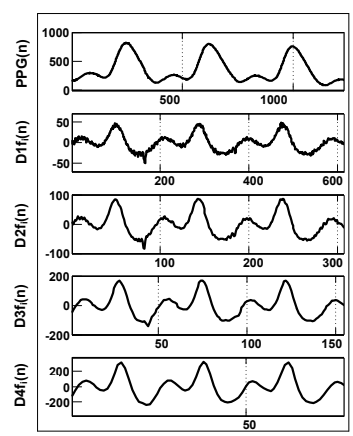

b)
Fig. 3. (a) ECG signal and detail coefficients from 4 levels of decomposition (b) PPG signal with first derivations of 4 levels of approximations.

\section{FPGA IMPLEMENTATION}

\section{A. System Architecture}

FPGA implementation of PTT meter follows the functional diagram in Fig. 4. It consists of several modules and runs by $50 \mathrm{MHz}$ clock. Analog signals $E C G(t)$ and PPG(t) are digitalized by 12 bit A/D convertor TLC2543 (TI) under sampling frequency of $f_{s}=800 \mathrm{~Hz}$. A/D converter is driven by $\mathrm{A} / \mathrm{D}$ controller module producing the digital samples $E C G[n][11 . .0]$ and $P P G[n][11 . .0]$. These samples are then fed to the ECG processing core and $P P G$ processing core respectively. ECG processing core produces QRS_pulse which indicates detected $\mathrm{R}$ wave while $P P G$ processing core detects maximal slope MS in PPG and generates $P P G \_$slope_pulse. QRS_pulse and $P P G$ slope_pulse are inputs to the PTT_calculator module which calculate $P T T I[10 . .0]$, an integer equivalent of $P T T$. PTTI[10..0] is sent out by UART controller.

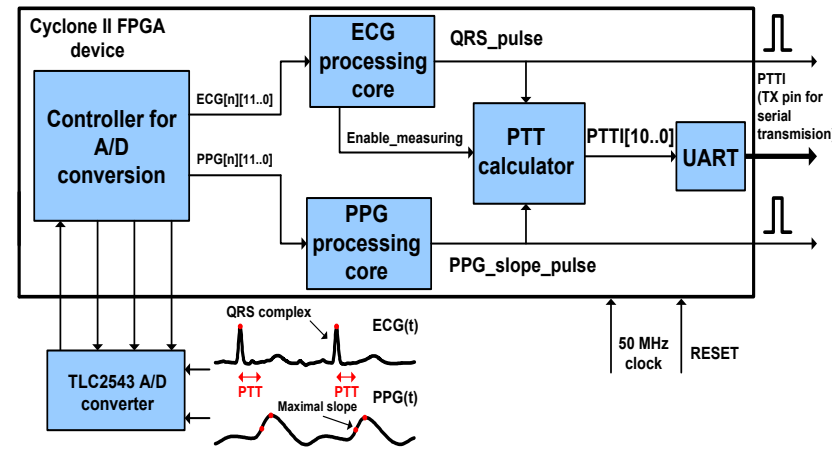

Fig. 4. Block diagram of PTT extraction by FPGA chip.

\section{B. QRS Detection}

The internal structure of the ECG processing core is given in Fig. 5. The digital samples $E C G[n][11 . .0]$ are fed to the Haar DWT block whose outputs are detail coefficients $D_{i}(n)[11 . .0]$ and approximation coefficients $A_{i}(n)[11 . .0]$, $\mathrm{i}=1 . .3$. The $D_{i}(n)[11 . .0]$, collected during $1 \mathrm{~s}$, from all levels, make arrays $D_{1}\left[N C_{1} \ldots 1\right][11 \ldots 0], D_{2}\left[N C_{2} \ldots 1\right][11 \ldots 0]$ and $D_{3}\left[N C_{3} \ldots 1\right][11 \ldots 0], N C_{i}=1 s^{*} f_{s} / 2^{i}, \quad N C_{1}=400, N C_{2}=200$ and $N_{3}=100$. Each of these arrays is then searched by module Local_min., Local_max. and Zero_cross. detector in order to locate characteristic points, local min, ZC and local max. In case of their occurrence in true sequence, the Pulse $i$ is generated. Condition for successful detection of QRS complex is appearance of at least one Pulse_ $i$ at the inputs of OR gate. Afterwards, the Final_pulse generator produces a $Q R S \_$pulse which is sent out of the ECG processing core.

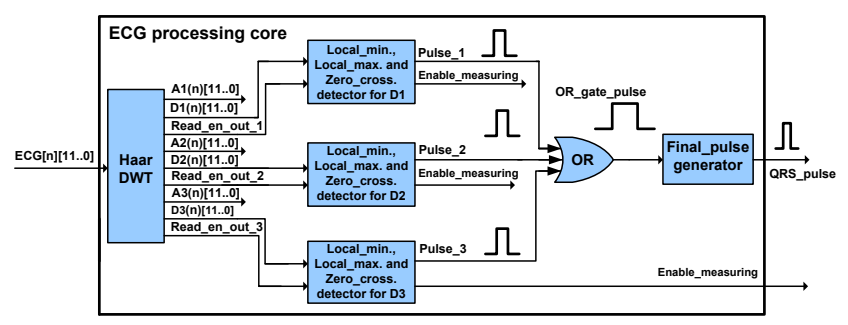

Fig. 5. Internal structure of ECG processing core. 
Each module Local min, Local max and Zero-cross. consists of 5 sub-modules: Local_min. detector; Local_max. detector; Zero_cross. detector; State machine for QRS recognition and Pulse generator, Fig. 6. Operation of Local_min. detector and Local_max. detector follows the logic from Fig. 7. They generate signals Min_detected and Max_detected.

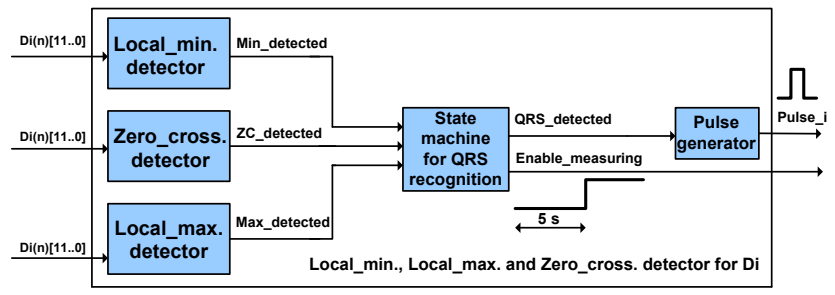

Fig. 6. QRS detection from $\mathrm{D}_{\mathrm{i}}(\mathrm{n})$ - structure.

Decision on local $\min / \max$ is based on adaptive thresholds $T_{p i}$ or $T_{n i}$ and the contents of three registers that contain three successive points $R 1=D_{i}(n)[11 \ldots 0], R 2=D_{i}(n-$ 1) [11..0] and $R 3=D_{i}(n-2)[11 . .0]$. The decision rules are as following: first, $I F\left((R 3<R 2)\right.$ and $\left(R 2>T_{p i}\right)$ and $\left.(R 1<R 2)\right)$ then Local_max $=R 2$ and second, $I F\left((R 3>R 2)\right.$ and $\left(R 2<T_{n j}\right)$ and $(R 1>R 2))$ then Local_min $=R 2$. ZC circuit works under principle $D_{i}(n)[11 \ldots 0]>0$ and $D_{i}(n-1)[11 \ldots 0]<0$ and only sign bits are compared.

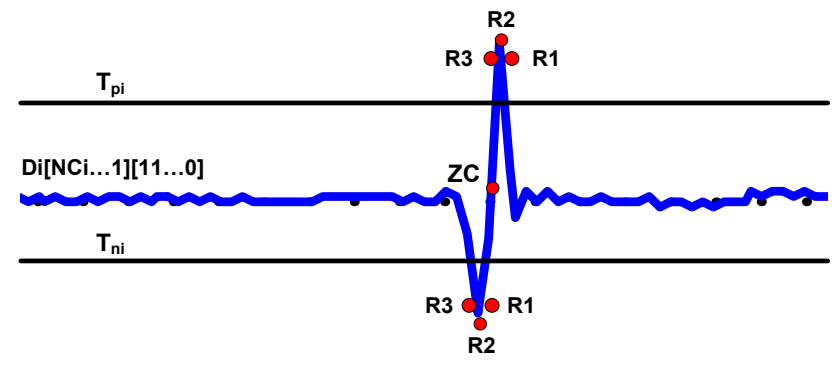

Fig. 7. Finding modulus maxima from $D_{i}(n)$.

QRS recognition is carried out using a state machine (SM) whose inputs are Min_detected, ZC_detected and Max_detected. The initial state lasts for 5 seconds and it serves for initial suiting of the adaptive thresholds, Fig 8. After initial adaptation, the signal Enable_measuring becomes high and process of QRS detection begins. Detection of QRS complex is successful if local max is detected after local $\min$ and ZC. Then QRS_detected synchronized output of SM is generated. QRS_detected from SM is fed to the Pulse_generator, which generates Pulse $i$ of $30 \mathrm{~ms}$ duration. Pulse_1, Pulse_2 and Pulse_3 are passing through the OR gate creating $O R \_g a t e \_p u l s e$ which is in fact the overlapped summation. $O R \_$gate pulse is fed to the Final_pulse generator which produces QRS_pulse of 20 ms duration. QRS_pulse happens only if between two sequent $O R \_$gate_pulses passed $200 \mathrm{~ms}$ that is considered as down physiological limit for $R R$ interval.

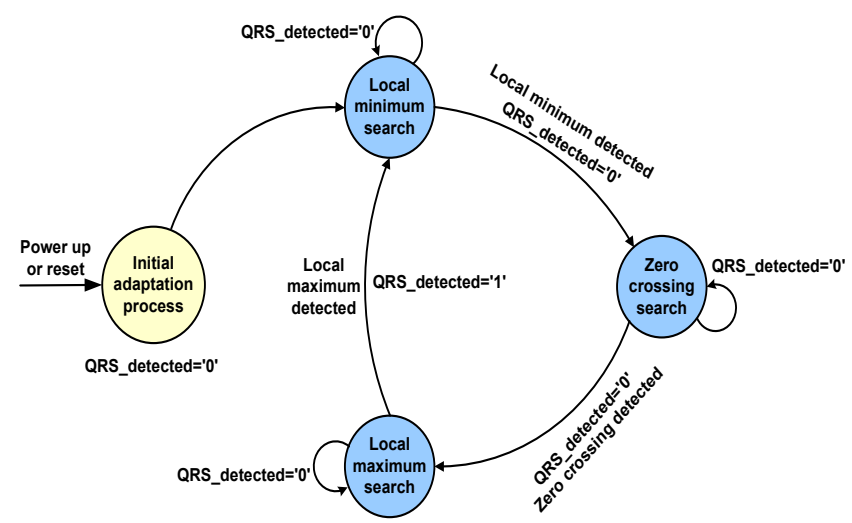

Fig. 8. State machine for $\mathrm{QRS}$ detection.

\section{PPG Slope Detection}

PPG samples, $P P G(n)[11 \ldots 0]$, are decomposed up to $3^{\text {rd }}$ level. Approximation coefficients $A_{1}(n)[11 . .0], A_{2}(n)[11 . .0]$ and $A_{3}(n)[11 \ldots 0]$ are fed to the derivative filter, producing $D 1 f_{i}(n)[11 . .0], D 2 f_{i}(n)[11 . .0]$ and $D 3 f_{i}(n)[11 . .0]$, and then to the Max. detector 1, Max. detector 2, and Max. detector 3, Fig. 9. The sequences of $D 1 f_{i}\left[N C_{1} . .1\right][11 . .0]$, $D 2 f_{i}\left[N C_{2 . .1}\right][11 . .0]$ and $D 3 f_{i}\left[N C_{3 . .1} .[11 . .0] \quad\left(N C_{I}=400\right.\right.$, $\left.N C_{2}=200, N C_{3}=100\right)$ are searched in order to find a local maxima by using thresholding technique. For each $\operatorname{Dif}_{i}(n)[11 . .0], T_{i}(\mathrm{i}=1 . .3)$ is an adaptive threshold.

Decision rule for local maxima detection for one $\operatorname{Dif}_{i}$ is: IF $\quad\left(\left(\operatorname{Dif}_{i}(n-2)[11 . .0]<\operatorname{Dif}_{i}(n-1)[11 . .0]\right) \quad\right.$ and $\quad\left(\operatorname{Dif}_{i}(n-\right.$ 1) $\left.[11 . .0]>T_{i}\right)$ and $\left.\left(\operatorname{Dif}_{i}(n)[11 . .0]<D i f_{i}(n-1)[11 . .0]\right)\right)$ then Local_max $=\operatorname{Dif}_{i}(n-1)[11 \ldots 0]$. When local maxima are detected, pulses Max_detected_1, Max_detected_2 and Max_detected_3 are generated and fed to state machine, SM for detection of maximal slope. This SM has initial state of adaptation of $T_{i}$ which lasts for five seconds. From detection state, after appearance of Max_detected_1, Max_detected_2 or Max_detected_3, the SM goes into PPG_slope_pulse generation state which lasts for $20 \mathrm{~ms}$. After this state, SM goes into standby mode for $180 \mathrm{~ms}$. From standby mode SM returns to detection state.

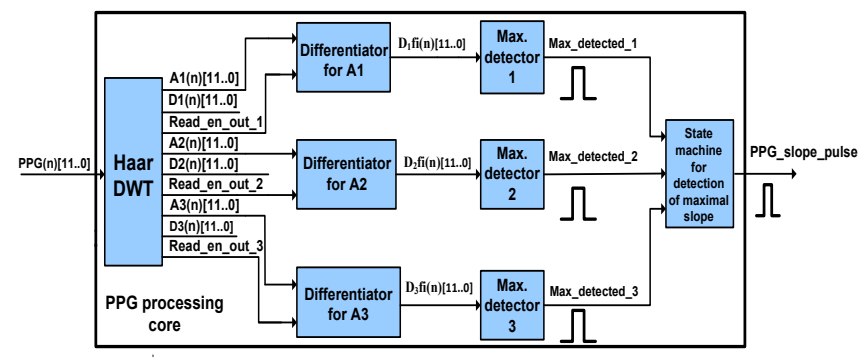

Fig. 9. Internal structure of PPG slope detection core.

\section{PTT calculation}

The circuit for PTT calculation consists of two counters (C1 and C2), one latch registers and control logic, Fig. 10. When QRS_pulse appears at input of PTT_calculator, counters $C 1$ and $C 2$ are enabled by control logic. $C 1$ is clocked by system clock, while $C 2$ is clocked each $1 \mathrm{~ms}$ using overflow (of.) of $\mathrm{Cl}$, and latched by 
$P P G \_$slope pulse. Output of $C 2, N_{m s}$ represents time elapsed from QRS_pulse appearance and it is latched to PTTI[10..0] each time the PPG_slope_pulse appears.

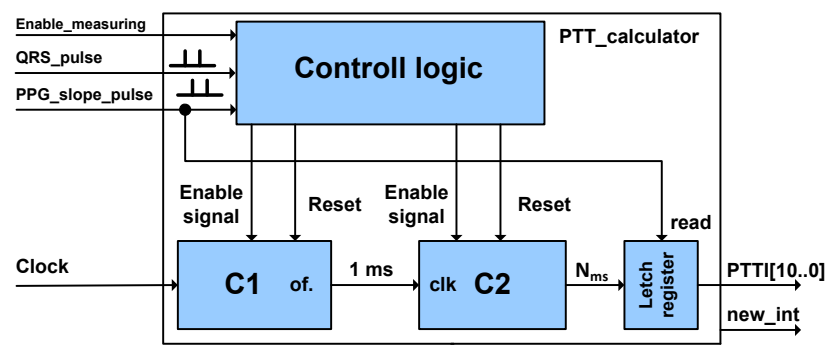

Fig. 10. PTT calculator structure.

\section{Testing And Results}

Overall system was developed in VHDL code and implemented in Cyclone II FPGA chip EP2C70F896C6N [5]. PTT intervals were transferred out via serial port, while QRS_pulses and $P P G \_$slope pulses were observed by oscilloscope.

The records from MIT-BIH Arrhythmia and MIMIC databases were used to test the system. Fig. 11 (a) shows the oscilograph of ECG signal and digital pulses, QRS_pulses, at output of the QRS detector, at the pin of the FPGA chip. Fig. 11 (b) shows the result for the detection of maximal slopes in PPG signals. QRS detector test was conducted on 10 records from MIT-BIH Arrhythmia database and it lasted for five hours. Testing was conducted on a total of 22031 beats, where there were 105 false detections and 424 missed detections. The average accuracy of the QRS detector is 97.53\%. PPG processing core was tested on records from MIMIC database. 5 x 10 -minute PPG records were examined, achieving average detection accuracy was $97.09 \%$.

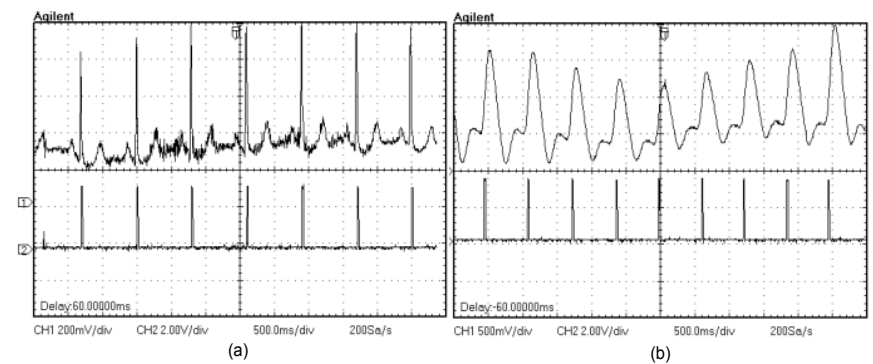

Fig. 11. (a) QRS detection from ECG (b) MS detection from PPG.
PTT calculation was tested on records from MIMIC database. ECG and PPG signals were processed in parallel in order to extract PTT intervals. Fig. 12 shows result for calculation of PTT intervals for MIMIC record no. 041 and confirms inverse correlation between PTT and systolic BP.

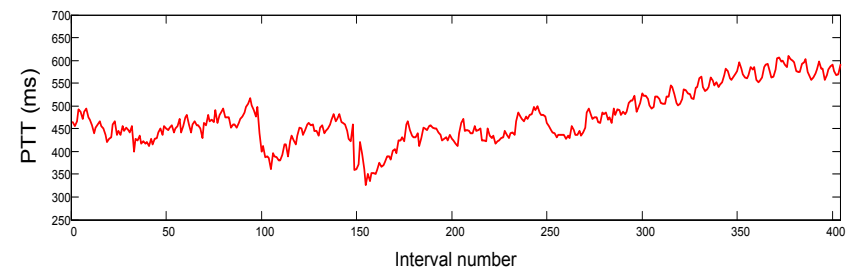

a)

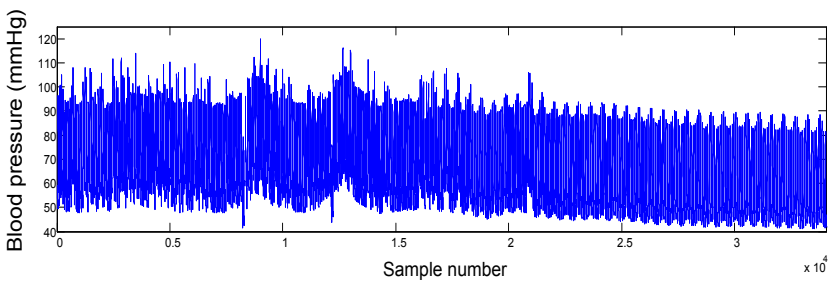

b)

Fig. 12. (a) PPT intervals and their correlation with (b) BP signal.

\section{CONCLUSION}

A system and methodology for real-time ECG and PPG processing and PTT interval calculation are presented. All tasks are implemented in a single FPGA chip. The system is achieving an accuracy of more than $97 \%$ for ECG and PPG signals. As such, it is ideal for embeded systems or wearable health care devices where cuff-less measuremnt of blood pressure is necessary.

\section{REFERENCES}

[1] WHO, "Cardiovascular diseases (CVDs)", Fact sheet N³17, March 2013, accessible http://www.who.int/mediacentre/factsheets/fs317/en/.

[2] M. H. Voelz, C. F. Babbs, J. D. Bourland, "Pulse Transit Time as an Indicator of Arterial Blood Pressure ", Psychophysiology, vol. 18, pp. 71-74, 1980.

[3] S.G. Mallat, "A theory for multiresolution signal decomposition: the wavelet representation", IEEE Trans. Pattern Analysis and Machine Intelligence, vol. 11, no. 7, pp. 674-693, 1989.

[4] R. Stojanović, D. Karadaglić, M. Mirković, D. Milošević, "A FPGA system for QRS complex detection based on integer wavelet transform", Measurement Science Review, vol. 11, issue 4, pp. 131$138,2011$.

[5] Altera Corporation, www.altera.com. 